\title{
A Novel Multi-Modal 3D Characterization System to Quantify Grain-Level Microstructural Features in Macro-Scale Volumes
}

\author{
Michael Uchic*, Michael Groeber*, Megna Shah**, Adam Shiveley*** \& Jonathan Spowart* \\ *Air Force Research Laboratory, Materials \& Manufacturing Directorate, Wright Patterson Air Force \\ Base, $\mathrm{OH} 45433$ \\ ** UES, Inc., 4401 North Dayton-Xenia Rd, Dayton, OH 45432 \\ *** Universal Technology Corporation, 1270 N Fairfield Road, Dayton, OH 45433
}

In order to rapidly quantify grain-level microstructures in structural alloys, a first-of-its-kind system is being assembled to characterize the 3D microstructure, local texture and local chemistry of macro-scale volumes ranging from $1 \mathrm{~mm}^{3}$ to $1 \mathrm{~cm}^{3}$. The system performs 3D characterization via serial sectioning, and has a unique combination of SEM-based analytical capabilities that are coupled to a bulk mechanical polishing system that can remove material with sub-micrometer precision. The initial application for this system is the microstructural quantification of nickel superalloys and titanium alloys, but the device is expected to be generically applicable to a diverse range of engineering materials.

The initial configuration of the multimodal system is comprised of three primary subsystems: (a) Tescan Vega 3 scanning electron microscope outfitted with a Bruker Quantax EDS and EBSD detectors for x-ray and crystallographic microanalysis, (b) UES RoboMet.3D.v2 robotic serial sectioning system for sequential material removal [1], and (c) 6-axis Mitsubishi RD12SVL robot with custom end-of-armtooling to perform sample exchanges between the other two subsystems. The incorporation of crystallographic [2] and chemical (spectral) [3] maps into 3D characterization systems has been previously realized on FIB-SEM instruments, however, these devices are only capable of interrogating miniature volumes, $<0.001 \mathrm{~mm}^{3}$. Importantly, the use of both EBSD and EDS data allow for more accurate and robust procedures to automatically classify key microstructural features such as grains or second phases compared to traditional image-based analysis [2,3]. Note that all SEM images and analytical maps generated by the system will be stored in a single MXA-formatted file, which is optimized for multi-modal 3D data archival [4].

The associated presentation will highlight the development of the multi-modal system, discuss methods used to automate the data collection process, and present initial results from the characterization of $\mathrm{Ni}$ superalloy microstructures.

[1] Spowart JE. Proc. Microscopy Microanalysis, Cambridge University Press, Cambridge, UK (2006) 90-91

[2] Groeber MA, Haley BK, Uchic MD, Dimiduk DM, Ghosh S. Materials Characterization 57 (2006) 259-273

[4] Kotula PG, Keenan MR, Michael JR. Microscopy \& Microanalysis 12 (2006) 36-48

[4] Jackson M, Simmons JP, De Graef M. Modeling and Simulation in Materials Science and Engineering 18 (2010) 065008

The authors would like to acknowledge the following people that have contributed to the development of this system: Luke Creachbaum, Petr Tichopádek, Filip Lopour, Tony Owens, Kent Walker, Allen Poe, and Scott Schoessow. 